\title{
Futuro de la Universidad tras la pandemia del COVID-19
}

\section{Future of the University after the pandemic COVID-19}

\author{
José Manuel Bautista-Vallejo ${ }^{a}$
}

\begin{abstract}
Resumen
El presente trabajo lleva a cabo un análisis prospectivo de la institución universitaria en relación a tres elementos fundamentales (tecnología, incertidumbre y competencias), los cuales dibujan un horizonte para el aprendizaje en este nivel terciario, en el marco complejo de la pandemia por COVID-19. La reflexión comienza con la pregunta de si, en la situación de pandemia actual, lo que está en juego es la civilización occidental. Pese a que la respuesta puede ser positiva o negativa, lo que sí parece claro es que en los próximos años la sociedad y las instituciones humanas que forman la misma es probable que inicien una revisión sobre sus presupuestos y forma de proceder. Esta revisión se viene considerando desde, por lo menos, mitad del siglo pasado. En concreto, la institución universitaria, que se ha visto impactada fuertemente por la pandemia, tendrá que tomar decisiones sobre su naturaleza y condición. El escenario postpandémico estará enmarcado por un desarrollo tecnológico sin precedentes, una incertidumbre galopante y la enseñanzaaprendizaje de competencias, tal vez de una manera diferente a cómo se aborda esta cuestión hoy. Es ahí donde cabe preguntarse por el futuro de la universidad.
\end{abstract}

Palabras clave: universidad, competencias, pandemia, aprendizaje.

\begin{abstract}
This work carries out a prospective analysis of the university institution in relation to three fundamental elements (technology, uncertainty and competences), which draw a horizon for learning at this tertiary level, in the complex framework of the COVID-19 pandemic 19. Thereflection begins with thequestion of whether, in the current pandemic situation, what is at stake is Western civilization. Although the answer may be positive or negative, what does seem clear is that in the coming years society and the human institutions that comprise it are likely to initiate a review of their budgets and how to proceed. This revision has been under consideration since at least half of the last century. Specifically, the university institution, which has been strongly impacted by the pandemic, will have to make decisions about its nature and condition. The post-pandemic
\end{abstract}

a Universidad de Huelva,
España.

Correspondencia a: bautista@uhu.es

Recibido:

10 de mayo de 2020

Aceptado:

24 de junio de 2020

Artículo publicado en acceso abierto bajo la Licencia Creative Commons.

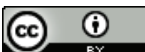

Cita:

Bautista-Vallejo, J. B. (2020). Futuro de la Universidad tras la pandemia del COVID-19. Kera Yvoty: reflexiones sobre la cuestión social, 5(número especial), 43-49. 
scenario will be framed by unprecedented technological development, rampant uncertainty and the teaching-learning of skills, perhaps in a different way than how this issue is approached today. This is where it is worth asking about the future of the university.

Keywords: university, skills, pandemic, learning.

\section{Introducción}

Hay quien afirma que, en la situación de pandemia actual, lo que está en juego es la civilización occidental. Se equivoque o no, en los próximos años sociedad e instituciones humanas tal vez inicien una revisión sobre sus presupuestos y forma de proceder. Esta puede ser la anunciada y esperada revisión del tercer milenio. Una de estas instituciones, la universitaria, que se ha visto impactada fuertemente, tendrá que tomar decisiones sobre su naturaleza y condición, en un marco de desarrollo tecnológico sin precedentes, incertidumbre galopante y marco competencial que aparece como incontestable.

En este escenario, ¿cuál es el futuro de la universidad?

\section{Nuevo escenario: desarrollo científico, incertidumbre y competencias}

El desarrollo científico y técnico en las últimas décadas ha sido vertiginoso. Esta suele ser una expresión recurrente. Si bien es cierto, la moneda en uso ha demostrado tener también otras caras, manifestadas por hechos cada día más claros: este avance tecnológico al mismo tiempo que puede ser calificado como sin precedentes, debe ser criticado como desigual, en un marco de profunda incertidumbre, ya no solo por el futuro, sino también por el presente.

Por otro lado, de forma transversal $y$, también, global, la instauración de un modelo de enseñanza y aprendizaje por competencias ha sido el instrumento adoptado para superar buena parte de los problemas identificados como tal, en una era donde la incertidumbre no nos ha abandonado. En él se han puesto todas las esperanzas, si bien pocos han percibido que el modelo por competencias era, claramente, una ideología más con todo lo que ello implica.

Estos tres elementos (tecnología, incertidumbre y competencias), dibujan el horizonte para el aprendizaje, incluida el de su industria. Sin embargo, pese a que la sociedad está cambiando y las personas se verán obligadas a renovar continuamente su estado cognitivo, es decir, a seguir aprendiendo, esta apreciación (sociedad del aprendizaje), aunque se escucha con frecuencia es bastante ambigua (Barnett, 2001).

Así pues, no puede negarse que la tecnología se ha desarrollado de forma exponencial, con grandes avances y a una velocidad de transformación de la realidad sin precedentes. Los conocimientos se acoplan, cambian y propagan en una imparable progresión. Esta masa enorme de conocimientos rebasa actualmente el poder de asimilación mental de las personas (Molsalve Lorente e al., 2020). La rapidez en la renovación de los conocimientos es de tal grado que, a menudo, se tiene la impresión de simplemente reaccionar ante nuevos estímulos, en un legítimo afán de no ceder al curso apresurado de los acontecimientos, más que de controlarlos y responder con plena conciencia ante ellos. En ese sentido, la evolución tecnológica e informática es, tal vez, el principal factor del cambio social de nuestra época (Tirado-Morueta et al., 2020).

Este avance tecnológico tiene ciertos momentos clave a lo largo del siglo XX. Un marco para entender su evolución e impacto requiere de la descripción de la modernidad y la postmodernidad. Así, aunque tras la Segunda Guerra Mundial asistimos al renacimiento de un optimismo pedagógico, tal vez propio de etapas 
postbélicas, la llamada modernidad se daría por terminada hacia mitad de los años 50 del siglo XX.

Probablemente lo que caracteriza a la modernidad sea el intento de superar mediante el racionalismo el escepticismo total en que desemboca el empirismo, pues el hombre moderno, ofuscado por la ciencia y de una manera especial por su formalismo matemático, piensa que puede escapar del escepticismo epistemológico y del relativismo moral, para lo cual funda tanto la ciencia como la moral únicamente en el poder de la razón.

Sin embargo, críticos como Susan Sontag, Ihab Hasan y, sobre todo, Leslie Fiedler comprobaron que la modernidad había concluido desde aproximadamente el año 1955. Los provocadores críticos culturales prometieron una nueva época de un "irracionalismo refrescante", una nueva sensibilidad que se despidió de una estética iluminista, política y a veces también de un notorio carácter pedagogizante (Bautista Vallejo, 2006).

Las alarmantes tendencias de las sociedades actuales hacia el narcisismo y el atomismo, el declive de la participación cívica, la creciente sensación deque todas las relaciones y compromisos son revocables, la diversificación, fragmentación y hasta acercamiento a su contexto de uso del conocimiento, y el crecimiento de actitudes cada vez más "instrumentales" hacia la naturaleza y la sociedad, son manifestaciones de un deslizamiento hacia el subjetivismo, al que es proclive la cultura y condición postmoderna (Lyotard, 1994). En este nuevo contexto, la inseguridad y la incertidumbre son rasgos definitorios.

Lo que se vio tocado de fondo fue, pues, las condiciones de certeza absoluta, a lo que se ha sumado la fragmentación del yo personal y el individualismo, conduciendo, así, al escepticismo epistemológico. Si bien esto, y dada la condición del ser humano como ser falible en un mundo cargado de dinamismo, la incertidumbre y la posibilidad de ignorancia son elementos consustanciales de ese mundo.
Pero, dadas ciertas condiciones éstas no tienen la última palabra. Hay una tendencia al equilibrio, no alcanzado del todo (Hayek, 2007) y a la posibilidad de desvelar el error y aprender en ese marco de incertidumbre. Hay una tendencia al equilibrio en medio de nuestra ignorancia (Zanotti, 2006), algo que Hayek (2007) llamaba "factor de aprendizaje", una capacidad intuitiva de aprendizaje a partir de nuestros errores y de estar alerta a las oportunidades en un mundo donde en condiciones de libertad se da el conocimiento disperso.

$\mathrm{Si}$ bien el conocimiento es un rasgo esencial de la sociedad moderna, la aparición de nuevos términos ayuda a comprender la nueva situación. Términos como intelecto, conocimientos, verdad, objetividad y disciplinas, van dejando paso a otros como competencias, resultados, habilidades y capacidad de transferencia. Ambos describen esa visión de la universidad que sirve a la cultura cognitiva o a la economía (Barnett, 2001).

El nuevo vocabulario, "que incluye términos como 'competencia', 'resultados de aprendizaje', 'acumulación de créditos' 'perfiles de aprendizaje' y se refiere a los estudiantes como 'productos', no solo es sintomático de los cambios internos del currículo de la educación superior, sino que además evidencia una reconfiguración del conocimiento que responde a las demandas contemporáneas. En una sociedad en la cual importan la adquisición inmediata de habilidades, la acción encaminada a efectos y la información, se producen cambios en el ámbito académico respecto de cuáles son los conocimientos importantes" (Barnett, 2001, p. 70).

Barnett (2001) critica este planteamiento porque si bien la idea de estudiante como producto responde a un procesodetransformacióndesdequeingresa hasta que egresa del sistema, en un marco de finalidades predeterminadas y de resultados uniformes, esto no es lo más adecuado para un proceso de interiorización personal, de imprevisibilidad y de incertidumbre, 
en donde se manifieste un currículum abierto. "Un currículum construido de esta manera no es un conjunto pre-armado de información y metodología al cual el estudiante se encuentra sujeto, sino más bien un marco relativamente flexible en el que los estudiantes pueden desarrollarse y del cual finalmente despegarán por su cuenta. Una educación superior es un proceso abierto y no una venta de productos predeterminados" (Barnett, 2001, p. 71).

\section{Conclusión: la pandemia del siglo XXI y el futuro de la universidad}

Vista la cuestión desde fuera de su núcleo, hay que tener en cuenta que la misma no es nada nueva, ni como hecho ni como reacción. En 2013, el politólogo Wallerstein había escrito sobre estas contingencias y predijo que a partir de 2020 tres elementos podrían afectar nuestro sistema mundo, como él lo denominaba, y sus instituciones: el cambio climático, las pandemias y la guerra mundial. Estamos en 2020.

Curiosamente, el discurso sobre el cambio climático se ha hecho global, con dudosas medidas para contrarrestar sus efectos, mínimas por parte de las instituciones universitarias (De la Rosa Ruiz et al., 2019). Al tiempo, la pandemia del COVID-19 recorre el planeta entero, con informes que se refieren a millones de infectados por el virus y una cifra elevada de muertos (Sohrabi et al., 2020), algo que, como vemos, ya ha afectado a la manera de enseñar y aprender de millones de profesores y estudiantes en todo el mundo.

El caso de las universidades de China, por ser el primero y afectar a millones de universitarios, ha sido muy significativo (Wang, Cheng et al., 2020), al que ha seguido el de decenas de países en todo el mundo (Sund, 2020).

El interés de buena parte de la pedagogía actual sobre esta metodología se basa en la relación directa con el impacto en las diversas formas de aprendizaje. Todas ellas son dependientes de la visión del aprendizaje que hemos desarrollado más arriba. En este sentido, la crisis actual de la pandemia por COVID-19 ha puesto de manifiesto la fragilidad de nuestro sistema universitario y su forma de "organizar" el aprendizaje, en varios sentidos.

Por un lado, la situación sobrevenida ha llevado a miles de instituciones universitarias en todo el mundo a una aceleración en las medidas de virtualización de la enseñanza y aprendizaje (Bouza Mora, 2020; Wang, Zhang et al., 2020). La respuesta está siendo desigual, si bien muchas universidades se han reconvertido de manera acelerada. El impacto que esto va a tener sobre el aprendizaje y la manera de aprender está, ciertamente, lleno de incógnitas.

La virtualización de la universidad, por tanto, va a afectar a cuatro elementos fundamentales. Por un lado, lo tecnológico y lo administrativoy, por otro, lo pedagógico y lo comunicativo, hasta generar una situación totalmente nueva, lo que habitualmente se define como un antes y un después.

Pese a lo que pueda decirse, la incertidumbre sigue regando las instituciones de todo el mundo, entre ellas las universitarias.

La universidad, así, está en el núcleo de la cuestión o, al menos, en el centro de algunas de las cuestiones. Se observan fenómenos como los de fragmentación, diversificación, instituciones alternativas que rivalizan con las universidades, aparición de nuevos lugares de producción del conocimiento, etc. Nada de esto es ajeno al espacio universitario. En algún sentido la universidad corre el peligro, incluso, de quedar desplazada por el giro protagonista en la aparición de nuevos fenómenos.

En esta situación, los cambios experimentados fuera de las instituciones educativas plantean nuevas exigencias a la educación $y$, por consiguiente, a la universidad. Tradicionalmente, a tenor de la lentitud de los cambios de la sociedad, la educación preparaba a las personas desde y para unas condiciones de vida que apenas variaban durante mucho tiempo. Pero, ¿qué 
ocurre cuando estos cambios se producen de forma exponencial?

Durante los últimos años, aspectos tales como el desarrollo de la creatividad, la capacidad de adaptación al cambio, aprender a aprender, la resolución de problemas, nuevas interpelaciones del alumnado, los cambios de paradigma en las respectivas disciplinas, etc., se han incorporado con fuerza como parte de las funciones de la universidad, pero más en el plano teórico que en el práctico, cuyo modus operandi no pareció cambiar (Bautista-Vallejo et al., 2019).

Cabe, entonces, la pregunta: ¿quées lo importante en la universidad? La respuesta es $\tan$ diversa que no solo ofrece dudas, también ofrece equívocos, puesto que cada programa tiene una respuesta diferente. Desde su fundación, la universidad era definida como el "ajuntamento" de profesores y estudiantes, institución que servía a tres fines: ofrecer y formar en una cultura de excelencia, enseñar una profesión e investigar (Bautista Vallejo, 2005). Con estos tres fines, la universidad ha atravesado los tiempos y los acontecimientos.

Pero todo acontecimiento, y esto es norma social, envía información que se recoge por las personas y las instituciones, que pueden ser vistos como organismos vivos, y se convierte en maestro interior. Este conocimiento puede permitir reaccionar, validar, interpretar lo acontecido, de tal manera que la incertidumbre no hunde toda iniciativa y libertad humana. Visto de esta forma, las instituciones humanas, como la universitaria, pueden ser diseñadas para crear bajo condiciones de incertidumbre extremas, y esto es fundamental también para salvaguardar la universidad de hoy.

Y, ¿qué hay del fracaso? Forma parte de los intentos humanos. Profesorado y alumnado no deben ser considerados como meros aficionados, con comportamientos de ejecución mecánica y actuaciones prescritas por otros. Por tanto, el nivel de compromiso debe estar a la base de todo componente cognitivo, emocional y comprensivo que implique el aprendizaje que en la universidad tiene lugar. Lo que ocurra en la universidad tiene que invadir el mundo de la vida.

¿Es posible predecir cuáles serán los cambios que seguirán a las experiencias de aprendizaje que se desarrollen en la universidad? Desde la visión de la competencia académica se dirá que sí. Herramientas como la transmisión de un programa y la repetición del mismo ayudan solo una parte a generar una respuesta diferente. ¿En qué sentido ha de ser diferente? En que realmente no podemos anticipar totalmente cuáles serán los cambios existenciales que se producirán en el alumnado tras su paso por la universidad. Sin embargo, es seguro que éstos van a existir (Barnett, 2001).

¿Cómo enfocar, entonces, el trabajo universitario? Ciertamente, el trabajo docente universitario se puede dar por satisfecho cuando el alumnado afirme al finalizar un curso: "este curso me ha cambiado", "ahora veo las cosas de manera diferente". No se trata solo de una afirmación sobre lo que piensa, es una afirmación sobre él o ella misma, no cambia solo su pensamiento, cambia también la persona.

Lo trágico es que la naturaleza de esta comprensión está al margen de lo que las competencias indican en sus estándares establecidos. Las competencias no ayudan al desarrollo de la comprensión. La comprensión puede expresarse en los estudiantes de múltiples formas y raras veces son éstas permitidas en las universidades.

La comprensión, ahora que la incertidumbre se hace más patente debido a la pandemia, puedealiarse con la autonomía y con la formación que, en este momento y no sabemos hasta cuándo, se verá mediada por máquinas y a distancia. Lo que llama la atención es que en el mayor experimento a nivel mundial jamás realizado, esta distancia, que es transaccional, puede arrojar, si seguimos a Michael Moore (1993), cuya revisión teórica (Shearer \& Park, 2019) define esta transacción como un hecho inmutable, algunos beneficios 
interesantes en relación a la comprensión y a ciertos descubrimientos personales, a la postre objetivos muy importantes para la institución universitaria: la esencia de este tipo de formación es la independencia del estudiante cuando hay menor diálogo y más distancia transaccional.

Es decir, cuando hay menor diálogo profesor-alumno y más estructura de un determinado curso o programa, hay más distancia transaccional y, por consiguiente, mayor autonomía. En la situación actual, esto es lo que permite explicar que buena parte del alumnado no esté aprendiendo lo que estrictamente viene en los programas, pero estén ganando desde su autonomía determinados niveles de comprensión y transformacióndesusestructurascognitivas con nuevas formas de interacción.

Volveríamos a tener, entonces, un sistema que, bien mirado, puede permitir más libertad para ganar mayor autenticidad del ser humano.

\section{Referencias}

Barnett, R. (2001). Los límites de la competencia. El conocimiento, la educación superior y la sociedad. Gedisa.

Bautista Vallejo, J. M. (2005). Universidad y Espacio Europeo en la encrucijada de la calidad. Hergué.

Bautista Vallejo, J. M. (2006). Educar en la posmodernidad. Descubrir personas y orientar su desarrollo. EUNED.

Bautista-Vallejo, J. M., Espigares-Pinazo, M. J., \& Hernández-Carrera, R. M. (2019). El EEES en la Universidad española como innovación y el papel del alumnado veinte años después. En: F. J. Hinojo-Lucena; I. Aznar-Díaz, \& M. P. Cáceres Reche (Eds.), Avances en recursos TIC e innovación educativa (pp. 139-151). Dykinson. https://doi. org/10.2307/j.ctv105bcf5.15

Bouza Mora, D. (2020). Instrucción: UNAVD-DISC-o03-2020. Modificación de las actividades académicas durante la situación de emergencia por COVID19 en la Universidad Nacional. Costa Rica:
Universidad Nacional.

De la Rosa Ruiz, D., Giménez Armentia, P., \& Maldonado, C. (2019). Educación para el desarrollo sostenible: el papel de la universidad en la Agenda 2030. Revista Prisma Social, 25, 179-202. http://hdl.handle.net/10641/1691

Gallagher, M. P. (1994). Responses to modernity. Cultures et Foi - Cultures and Faith - Culturas y Fe, II(4), 282-292.

Hayek, F. A. (2007). Nuevos estudios de filosofía, política, economía e historia de las ideas. Unión Editorial.

Lyotard, J. F. (1994). La condición postmoderna. Cátedra.

Molsalve Lorente, L., Pardo Baldoví, M. I., \& Vidal Esteve, M. I. (Eds.). (2020). Pedagogía y cambios culturales en el siglo XXI: Repensando la educación. Octaedro.

Moore, M. G. (1993). Theory of transactional distance. En: D. Keegan (Ed.), Theoretical principles of distance education (vol. 1, pp. 22-38). Routledge.

Shearer, R. L., \& Park, E. (2019). The Theory of Transactional Distance. En: I. Jung (eds), Open and Distance Education Theory Revisited. SpringerBriefs in Education. Springer.

Sohrabi, C., Alsafi, Z., O'Neill, N., Khan, M., Kerwan, A., Al-Jabir, A., Iosifidis, C., \& Agha, R. (2020). World Health Organization declares global emergency: A review of the 2019 novel coronavirus (COVID-19). International Journal of Surgery, 76, 71-76. https:// doi.org/10.1016/j.ijsu.2020.02.034

Sund, K. J. (2020). Suggestions for Moving Teaching Rapidly Online in the Face of the Corona Crisis. Roskilde University.

Tirado-Morueta, R., García-Ruiz, R., Hernando-Gómez, Á., ContrerasPulido, P., \& Aguaded-Gómez, J. I. (2020). From Internet access to problematic use: multigroup analysis of push and pull factors. Behaviour $\mathcal{E}$ Information Technology, (8), 1-15. https://doi.org/10.1080/o14492 9X.2020.1751289 
Wang, C., Cheng, Z., Yue, X. G., \& McAleer, M. (2020). Risk Management of COVID-19 by Universities in China. Journal of Risk and Financial Management, 13(2), 1-6. https://doi. org/10.3390/jrfm13020036

Wang, G., Zhang, Y., Zhao, J., Zhang, J., \& Jiang, F. (2020). Mitigate the effects of home confinement on children during the COVID-19 outbreak. The Lancet, 1-2. https://doi.org/10.1016/So1406736(20)30547-X
Zanotti, G. J. (2006). Hacia un liberalismo clásico como la defensa de la intimidad personal. Doxa, 4, 233-253.

\section{Sobre el Autor}

José M. Bautista-Vallejo

Doctor Europeo en Psicopedagogía. Profesor de la Universidad de Huelva (España), ha enseñado en Universidades de Paraguay, Brasil, Argentina y Costa Rica. 